\title{
Performance Assessment of Linearized OPF-based Distributed Real-time Predictive Control
}

\author{
Rahul Gupta $^{1}$, Fabrizio Sossan ${ }^{1,2}$, Mario Paolone ${ }^{1}$ \\ ${ }^{1}$ Distributed Electrical Systems Laboratory, EPFL, Switzerland \\ ${ }^{2}$ PERSEE, Mines ParisTech, France
}

\begin{abstract}
We consider the problem of controlling heterogeneous controllable resources of a distribution network with the objective of achieving a certain power flow at the grid connection point while respecting local grid constraints. The problem is formulated as a model predictive control (MPC), where a linearized grid model, to retain convexity, based on sensitivity coefficients (SCs) is used to model the grid constraints. We consider and compare the modelling performance of three different update policies for the SCs: when they are updated once per day considering static injections, updated once per day considering point prediction of the nodal injections, and recursively estimated using on-line measurements. Simulations are performed considering the CIGRÉ low voltage benchmark network. Performance is evaluated in terms of convergence speed, tracking error, and constraints modeling errors. Further, we perform a sensitivity analysis on the dominant model w.r.t. the length of the predictive horizon and number of controllable units.
\end{abstract}

Index Terms-Distributed control, energy storage, photovoltaic (PV), linear optimal power flow, sensitivity coefficients.

\section{INTRODUCTION}

Dispatching distribution networks to track a predefined dispatch plan at the grid connection point (GCP) has been proposed in the literature as a way to achieve the coordination between transmission networks and distribution systems (e.g. [1], [2]) and avoid the activation of expensive reserve. The work in [3] proposed a control scheme based on the alternating direction method of multipliers (ADMM) to coordinate the operations of heterogeneous resources and track the dispatch plan. The formulation in [3] was experimentally validated with a battery energy storage systems (BESS) and a curtailable PV facility, however, without considering the grid constraints on nodal voltages and branch currents.

Including the exact grid constraints in the optimization problem leads to a non-convex problem known as optimal power flow. Its convexification, to increase tractability, is typically achieved by relaxing non-convex constraints or by linearizing them. Authors in [4] proposed a convex relaxation, which works properly for a class of networks, but without considering shunt elements, and ampacity constraints. An exact convex OPF, that includes both the shunt elements and line ampacity constraints, was proposed in [5]. However, both of them do not apply to meshed networks. A linearization method based on an iterative approach was described in [6], and in [7] applied to distribution networks. However, it can only be applied to networks without shunt elements. An alternative linearization method that can be applied to both radial and meshed networks and accounts for shunt elements too is using sensitivity coefficients (SCs) [8].

In this work, we use voltage, current and loss SCs to develop linearized grid models. In particular, we report three different ways to use SCs. Further, we utilize these linearized grid models for developing a real-time MPC control framework and compare their performance in terms of convergence speed, optimality, and constraint modeling errors w.r.t. the exact AC power flow model. In summary, the main contributions of this paper are i) inclusion of the grid model into the formulation of [3], ii) generalization of the distributed MPC formulation of [3] for any generic controllable unit, and iii) comparative assessment of different linear grid models for the MPC formulation.

The paper is organized as follows: Section II presents the description on the grid models, Section III describes the proposed control formulation, decomposition into distributed optimization, and resources' optimization models, Section IV presents the simulation results, and Section V summarizes the contributions of this paper and states the conclusions.

\section{GRID MODEL}

We consider a generic distribution network (meshed or radial) consisting of $n_{b}$ nodes and $n_{l}$ branches. Let vectors $\mathbf{v} \in \mathbb{R}^{\left(n_{b}-1\right)}$ and $\mathbf{i} \in \mathbb{R}^{n_{l}}$ represent nodal voltages magnitudes and branch currents magnitudes, respectively, and $\mathbf{p} \in \mathbb{R}^{\left(n_{b}-1\right)}$ and $\mathbf{q} \in \mathbb{R}^{\left(n_{b}-1\right)}$ the nodal active and reactive controllable injections for all nodes except the slack node. Scalars $p^{l}, q^{l} \in \mathbb{R}$ are the total active and reactive transmission losses as seen at the grid connection point. We assume the following hypothesis i) the system is in steady-state and can be modeled by phasors, which is able to track small power-dynamics, ii) the nodes are PQ nodes, and the nodal injections are not voltage dependent. The linearized nodal voltages, branch currents and losses can be expressed as:

$$
\begin{array}{r}
\mathbf{v}=\mathbf{A}^{\mathbf{v}}\left[\begin{array}{l}
\mathbf{p} \\
\mathbf{q}
\end{array}\right]+\mathbf{b}^{v} \\
\mathbf{i}=\mathbf{A}^{\mathbf{i}}\left[\begin{array}{l}
\mathbf{p} \\
\mathbf{q}
\end{array}\right]+\mathbf{b}^{i} \\
{\left[\begin{array}{l}
p^{l} \\
q^{l}
\end{array}\right]=\mathbf{A}^{l}\left[\begin{array}{l}
\mathbf{p} \\
\mathbf{q}
\end{array}\right]+\mathbf{b}^{l},}
\end{array}
$$

where $\mathbf{A}^{\mathbf{v}} \in \mathbb{R}^{\left(n_{b}-1\right) \times 2\left(n_{b}-1\right)}, \mathbf{A}^{\mathbf{i}} \in \mathbb{R}^{n_{l} \times 2\left(n_{b}-1\right)}$, and $\mathbf{A}^{l} \in$ $\mathbb{R}^{2 \times 2\left(n_{b}-1\right)}$ and $\mathbf{b}^{\mathbf{v}} \in \mathbb{R}^{\left(n_{b}-1\right)}, \mathbf{b}^{\mathbf{i}} \in \mathbb{R}^{n_{l}}$, and $\mathbf{b}^{l} \in \mathbb{R}^{2}$ are linear transformation matrices, and vectors respectively. In this 
work, these linear transformation parameters are determined using the method of [8] that allows to uniquely compute the $\mathrm{SCs}$ as a function of the grid topology and grid state. The method involves solving a load-flow and a systems of linear equations (identified by the function $\xi$ hereafter) presented in [8], which has a unique solution for every operating point when the load-flow Jacobian is locally invertible. (refer to Theorem 8.1 in [9]).

The linear model (1) is static, in the sense that the values of the matrices at time $t$ are computed based on the former knowledge of all the nodal injections at time $t$. In practical grid applications, like real-time control and scheduling of power systems, which typically require to compute the control actions for future time intervals, determining the linear transformation parameters entails the use of point predictions of the nodal injections, with a look-ahead time that depends on the length of the control horizon. From this standpoint, we can envisage three policies to compute and update the linear transformation parameters (LTPs).

Let the index $\underline{t}$ denote the current time index, $T$ the number of intervals in the control horizon (e.g., at time-resolution $\Delta t$ $=5$ minute), $t=\underline{t}, \underline{t}+1, \ldots, T$ the time indices spanning from the time $t \Delta t$ to the end of the control horizon $T \Delta t$, and $\mathbf{p}_{t}^{\text {unc }} \in \mathbb{R}^{\left(n_{b}-1\right)}$, and $\mathbf{q}_{t}^{\text {unc }} \in \mathbb{R}^{\left(n_{b}-1\right)}$ the uncontrollable active and reactive power nodal injections (obtained by measurement for the time $\underline{t} \Delta t$, and by forecasts for subsequent intervals $t \Delta t$ ). The three update policies are:

1) the SCs are computed using a single operating point and kept fixed throughout the control horizon; $\mathbf{A}_{t}$ and $\mathbf{b}_{t}$ can be expressed as ${ }^{1,2}$ :

$$
\begin{aligned}
& \mathbf{A}_{t}^{\mathbf{v}}=\xi\left(\widehat{\mathbf{p}}_{\underline{t}}+\mathbf{p}_{\underline{t}}^{\text {unc }}, \widehat{\mathbf{q}}_{\underline{t}}+\mathbf{q}_{\underline{t}}^{\text {unc }}\right) \\
& \mathbf{b}_{t}^{\mathbf{v}}=-\mathbf{A}_{t}^{\mathbf{v}}\left[\begin{array}{l}
\widehat{\mathbf{p}}_{\underline{t}}+\mathbf{p}_{\underline{t}}^{\text {unc }}-\mathbf{p}_{t}^{\text {unc }} \\
\widehat{\mathbf{q}}_{\underline{t}}+\mathbf{q}_{\underline{t}}^{\text {unc }}-\mathbf{q}_{t}^{\text {unc }}
\end{array}\right]+\widehat{\mathbf{v}}_{\underline{t}} ;
\end{aligned}
$$

2) the SCs are estimated using the point-predictions of load and generation for each time interval $t ; \mathbf{A}_{t}$, and $\mathbf{b}_{t}$ can be expressed as:

$$
\begin{aligned}
& \mathbf{A}_{t}^{\mathbf{v}}=\xi\left(\mathbf{p}_{t}^{\text {unc }}, \mathbf{q}_{t}^{\text {unc }}\right) \\
& \mathbf{b}_{t}^{\mathbf{v}}=\widehat{\mathbf{v}}_{t} ;
\end{aligned}
$$

3) the SCs are updated based on the trajectory of the control action optimized during the previous time interval. Also, the last power set-point is propagated to the next time interval; $\mathbf{A}_{t}$, and $\mathbf{b}_{t}$ can be expressed as:

$$
\begin{aligned}
& \mathbf{A}_{t}^{\mathbf{v}}=\xi\left(\widehat{\mathbf{p}}_{t-1}+\mathbf{p}_{t-1}^{\text {unc }}, \widehat{\mathbf{q}}_{t-1}+\mathbf{q}_{t-1}^{\text {unc }}\right) \\
& \mathbf{b}_{t}^{\mathbf{v}}=-\mathbf{A}_{t}^{\mathbf{v}}\left[\begin{array}{l}
\widehat{\mathbf{p}}_{t-1}+\mathbf{p}_{t-1}^{\text {unc }}-\mathbf{p}_{t}^{\text {unc }} \\
\widehat{\mathbf{q}}_{t-1}+\mathbf{q}_{t-1}^{\text {unc }}-\mathbf{q}_{t}^{\text {unc }}
\end{array}\right]+\widehat{\mathbf{v}}_{t-1} .
\end{aligned}
$$

Since the SCs are constant, the first policy may fail in respecting the voltage and current constraints in cases of sudden power variations along the day. The second and third

\footnotetext{
${ }^{1}$ For the sake of brevity, LTPs for voltage model are only presented, the same procedure can be followed for current, and loss models.

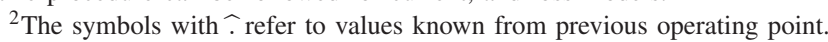

policies use dynamic SCs with updated information, however they require the availability of point predictions. To verify which among the three policies perform better, we perform in Sec. IV a detailed assessment of their performance.

\section{CONTROL FRAMEWORK}

We consider a distribution grid interfacing a cluster of controllable units and uncontrollable prosumption that is dispatched at its GCP according to a pre-determined dispatch plan. The dispatch plan, computed as in [2], is an optimal power consumption trajectory over a certain horizon (e.g., 5-minute resolution for the next 24 hours) computed before operation (e.g., the day-ahead). It is the sum of two elements: i) point predictions of the aggregated uncontrollable prosumption, and, ii) offset profile of each flexible resource, responsible to embed into the dispatch plan the energy demand required to restore optimal levels of flexibility in the controllable resources. The objective of the control is to coordinate the action of the controllable resources to track the dispatch plan on a 5-minute basis while respecting constraints of all resources as well as those of the grid (this last modelled by using the linear grid models discussed above). In the following, we first formulate the control problem in a centralized manner, and, then we show how it can be decomposed by leveraging on the ADMM-technique [10].

\section{A. Centralized model predictive control}

We consider $r=1, \ldots, R$ controllable resources connected to the grid, which can participate in tracking the dispatch plan. Let $\widehat{p}_{t}^{\text {disp }}$ be the dispatch plan set-point, the $q_{t}^{\text {gcp }}$ the decision variable for the reactive power at the GCP at time $t$, $\boldsymbol{x}_{r}$ the decision vector (active and reactive power set-points) for resource $r$, where $\boldsymbol{x}_{r}=\left[x_{r, \underline{t}+1}, \ldots, x_{r, T}\right]$ is referring to the power set-points from next time interval till end of the horizon $T \Delta t$. Here, $x_{r, t}=\left[p_{r, t}, q_{r, t}\right]$, where $p_{r, t}$, and $q_{r, t}$ are elements of nodal power generation vectors $\mathbf{p}_{t}$, and $\mathbf{q}_{t}$ respectively. The problem can be formulated as to minimize the sum of operation costs of different resources, $f_{r}\left(x_{r, t}\right)$, as:

$$
\underset{\boldsymbol{x}_{1}, \ldots, \boldsymbol{x}_{R}}{\operatorname{minimize}} \sum_{r=1}^{R} \sum_{t=\underline{t}+1}^{T} f_{r}\left(x_{r, t}\right) \text {. }
$$

subject to:

$$
\begin{array}{ll}
\sum_{r=1}^{R} p_{r, t}+\mathbf{1}^{T} \mathbf{p}_{t}^{\text {unc }}+p_{t}^{l}=\widehat{p}_{t}^{\text {disp }} & t=\underline{t}+1, \ldots, T \\
\sum_{r=1}^{R} q_{r, t}+\mathbf{1}^{T} \mathbf{q}_{t}^{\text {unc }}+q_{t}^{l}=q_{t}^{\text {gcp }} & t=\underline{t}+1, \ldots, T \\
\left|q_{t}^{\text {gcp }}\right| \leq \frac{\left|\hat{p}_{t}^{\text {disp }}\right|}{\tan \left(\pi / 2-\theta_{m}\right)} & t=\underline{t}+1, \ldots, T \\
\Phi_{r}\left(\boldsymbol{x}_{r}\right) \leq 0 & r=1, \ldots, R \\
v^{\min } \leq \mathbf{v}_{t} \leq v^{\max } & t=\underline{t}+1, \ldots, T \\
0 \leq \mathbf{i}_{t} \leq \mathbf{i}^{\text {max }} & t=\underline{t}+1, \ldots, T \\
\text { Grid constraints }(1) & t=\underline{t}+1, \ldots, T .
\end{array}
$$


Here, eq. (5b) enforces the controllable units to track dispatch plan throughout the control horizon. Eq. (5c), (5d) are the constraints on the reactive power at the GCP: it is defined by the intended power factor at the GCP, represented by $\cos \left(\theta_{m}\right)$. Eq. (5e) represents the constraints of individual resources, where $\Phi_{r}$ represents the constraint set for each resource $r$. Eq. (5f) and (5g) express the limits on the nodal voltages $\left[v^{\min }, v^{\max }\right]$ and branch currents $\left[0, \mathbf{i}^{\max }\right]$ respectively. The formulation in (5) is convex since $f_{r}$, and the resource constraints (5e) are convex in $\boldsymbol{x}_{r}$, and other constraints are linear. For brevity, the constraints $(5 c)-(5 h)$ are denoted by $\Psi\left(\boldsymbol{x}_{1}, \ldots \boldsymbol{x}_{R}\right) \leq 0$ in the following.

\section{B. From centralized to distributed}

Following the method described in [3], we introduce a barrier function $g$ with zero cost when the tracking error $(5 b)$ is respected and infinity otherwise:

$$
g\left(x_{1, t}, \ldots, x_{R, t}\right)= \begin{cases}0 & (5 \mathrm{~b}) \text { is respected } \\ \infty & \text { otherwise. }\end{cases}
$$

Let $\boldsymbol{z}_{r}$ be the auxiliary variables to copy the behaviour of original variables $\boldsymbol{x}_{r}$, the so-called copied variables. We can reformulate the optimization problem (5) as:

$$
\underset{\substack{\boldsymbol{x}_{1}, \ldots \boldsymbol{x}_{R} \\ \boldsymbol{z}_{1}, \ldots \boldsymbol{z}_{R}}}{\operatorname{minimize}} \sum_{t=\underline{t}+1}^{T}\left\{\sum_{r=1}^{R} f_{r}\left(x_{r, t}\right)+g\left(z_{1, t}, \ldots, z_{R, t}\right)\right\}
$$

subject to:

$$
\begin{array}{ll}
\boldsymbol{x}_{r}=\boldsymbol{z}_{r} & r=1, \ldots, R \\
\Psi\left(\boldsymbol{z}_{1}, \ldots, \boldsymbol{z}_{R}\right) \leq 0 . &
\end{array}
$$

The problem in (7) is a standard sharing problem and separable in $\boldsymbol{x}_{r}$. It can be solved in a distributed manner by each resources; then, the solutions from each resource can be sent to the aggregator that accounts for the global constraints and objectives. The set of constraints (7b) can be moved into the cost function by using a sequence of Lagrangian multipliers, denoted by $\boldsymbol{y}_{r}$. The augmented Lagrangian can be written as:

$$
\begin{aligned}
L_{\rho} & =\sum_{t=\underline{t}+1}^{T}\left\{\sum_{r=1}^{R} f_{r}\left(x_{r, t}\right)+g\left(z_{1, t}, \ldots, z_{R, t}\right)\right\}+ \\
& +\frac{\rho}{2} \sum_{r=1}^{R}\left(\left\|\boldsymbol{x}_{r}-\boldsymbol{z}_{r}\right\|_{2}^{2}\right)+\sum_{r=1}^{R} \boldsymbol{y}_{r}^{T}\left(\boldsymbol{x}_{r}-\boldsymbol{z}_{r}\right) .
\end{aligned}
$$

Let $\boldsymbol{u}_{r}=\boldsymbol{y}_{r} / \rho$ be the scaled dual variable, $\rho$ being the penalty parameter, the above problem can be solved in following three iterative steps using the scaled-ADMM sharing problem [10]:

1) Original variables update:

$$
\boldsymbol{x}_{r}^{k+1}:=\underset{\boldsymbol{x}_{r}}{\arg \min }\left\{\sum_{t=\underline{t}+1}^{T} f_{r}\left(x_{r, t}\right)+\frac{\rho}{2}\left\|\boldsymbol{x}_{r}-\boldsymbol{z}_{r}^{k}+\boldsymbol{u}_{r}^{k}\right\|_{2}^{2}\right\}
$$

subject to:

$$
\Phi_{r}\left(x_{r, t}\right) \leq 0 \quad t=\underline{t}+1, \ldots, T .
$$

2) Copied variables update:

$$
\begin{aligned}
{\left[\boldsymbol{z}_{1}^{k+1}, \ldots, \boldsymbol{z}_{R}^{k+1}\right]:=} & \underset{\boldsymbol{z}_{1} \ldots \boldsymbol{z}_{R}}{\arg \min }\left\{\sum_{t=\underline{t}+1}^{T}\left\{g\left(z_{1, t}, \ldots, z_{R, t}\right)\right\}+\right. \\
& \left.\frac{\rho}{2} \sum_{r=1}^{R}\left\|\boldsymbol{x}_{r}^{k+1}-\boldsymbol{z}_{r}+\boldsymbol{u}_{r}^{k}\right\|_{2}^{2}\right\}
\end{aligned}
$$

subject to:

$$
\Psi\left(z_{1}, \ldots, z_{R}\right) \leq 0 .
$$

3) Dual variable updates:

$$
\boldsymbol{u}_{r}^{k+1}=\boldsymbol{u}_{r}^{k}+\boldsymbol{x}_{r}^{k+1}-\boldsymbol{z}_{r}^{k+1} \quad r=1, \ldots, R .
$$

Here, $k$ refers to the iteration index of ADMM. The original variables $\boldsymbol{x}_{r}$ in (9) is computed in parallel for each resource, $r=1, \ldots, R$. The updates of the copied variables $\boldsymbol{z}_{r}$ for $r=1, \ldots, R$ in (10), require collecting the local solutions from each resource, and it is solved by the aggregator. Also, the the dual variables in (11), $\boldsymbol{u}_{r}$ are updated by the aggregator. Then, the updated solutions of the copied and dual updated are disseminated to the resources. Eq. (9), (10), and (11) are solved till convergence criteria is met, i.e., when the primal and dual residual norms [10] reduce below a tolerance limit. For the penalty parameter $\rho$, we follow a self-adaptive approach as described in [10], [11].

\section{Application to a distribution system with BESS and PV}

We provide a practical example on how the ADMM formulation is applied to flexible resources such as a BESS unit, and a controllable PV plant.

1) BESS: the objective is to compute power set-points while obeying physical limits on the power rating and reservoir size. We account for BESS losses by integrating its equivalent series resistance into the network admittance matrix using the method described in [12]. Let the series $x_{b, t}=\left[p_{b, t}, q_{b, t}\right]$ be the decision variables for active and reactive power, where $x_{b, t}$ is an element of $\boldsymbol{x}_{b}$, the BESS decision problem is the following feasibility problem:

$$
\underset{\boldsymbol{x}_{b}}{\operatorname{minimize}} \sum_{t=\underline{t}+1}^{T} 1
$$

subject to:

$$
\begin{array}{ll}
\operatorname{SOE}_{t}=\mathrm{SOE}_{t-1}-p_{b, t} T_{s} & t=\underline{t}+1, \ldots, T \\
0 \leq\left(\left(p_{b, t}\right)^{2}+\left(q_{b, t}\right)^{2}\right) \leq\left(P_{\text {max }}^{b}\right)^{2} & t=\underline{t}+1, \ldots, T \\
a E_{\text {max }}^{b} \leq \mathrm{SOE}_{t} \leq(1-a) E_{\text {max }}^{b} & t=\underline{t}+1, \ldots, T
\end{array}
$$

where, $\mathrm{SOE}_{t}$ is the BESS state-of-energy, $T_{s}$ is the sampling time (300 sec in this case), $P_{\max }^{b}$, and $E_{\max }^{b}$ are the power and reservoir capacities respectively, and $0 \leq a<0.5$ is a fixed parameter to specify a margin on SOE limits. The constraint (12c) is to restrict the battery's apparent power within its fourquadrant converter capability. 
2) $P V$ : the objective is to minimize the total curtailments and operate at near-unity power factor. Let $x_{g, t}=\left[p_{g, t}, q_{g, t}\right]$ be the decision variables for active and reactive power, where $x_{g, t}$ is an element of $\boldsymbol{x}_{g}$. The PV problem is:

$$
\underset{\boldsymbol{x}_{g}}{\operatorname{minimize}} \sum_{t=\underline{t}+1}^{T}\left\{\left(p_{g, t}-\widehat{p}_{g, t}\right)^{2}+q_{g, t}^{2}\right\}
$$

subject to:

$$
\begin{array}{ll}
0 \leq\left(p_{g, t}\right)^{2}+\left(q_{g, t}\right)^{2} \leq\left(S_{\max }^{g}\right)^{2} & t=\underline{t}+1, \ldots, T \\
0 \leq p_{g, t} \leq \widehat{p}_{g, t} & t=\underline{t}+1, \ldots, T
\end{array}
$$

where, $S_{\max }^{g}$ is the rated power of the PV power converter, $\widehat{p}_{g, t}$ is the maximum power point (MPP) forecast of PV generation. Eq. (13b) and (13c) represent constraints on the active and reactive power respectively.

\section{Simulation and Results}

\section{A. Simulation setup, and input data}

1) Setup: The MPC scheme is simulated for the CIGRÉ low voltage benchmark network [13], i.e., a three-phase $0.4 \mathrm{kV} / 400 \mathrm{kVA}$, an 18-node system shown in Fig. 1. The nominal values of the uncontrollable nodal demands are shown in Table I. We refer to the setup described in Section III with physical characteristic of the resources as in Table I.

2) Demand and PV data: We simulate for a weekend (day 1 , and day 2), and a working day (day 3). The demand and PV data are collected from the experimental measurements described in [14]. They refer to 4 buildings in the electrical department at EPFL. Fig. 2a shows the prosumption at the GCP for the 3 days. The dispatch plan is computed using the procedure described in [2], and it is shown in Fig. 2b. The PV forecast for three days is shown in Fig. 2c.

TABLE I

NOMINAL DEMANDS AND CONTROLLABLE UNITS

\begin{tabular}{|c|c|c|c|}
\hline node Id & Demand (kVA) & pf & Resource (rating) \\
\hline \hline $1,11,16,18$ & $200,15,55,47$ & 0.95 & - \\
15 & 52 & 0.95 & PV $(60 \mathrm{kWp})$ \\
17 & 35 & 0.95 & BESS $(500 \mathrm{kWh} / 300 \mathrm{~kW})$ \\
\hline
\end{tabular}

\section{B. Comparison of MPC with different linear grid models}

1) Performance comparison: The metrics to measure the control performance of above linear grid models applied to the distributed MPC scheme are:

- Convergence speed of ADMM: it is measured in time and number of iterations, both expressed in terms of their mean, max and min values;

- Tracking error of the dispatch signal: it is the error between the pre-defined dispatch plan and the net prosumption after MPC. We show the RMSE (root mean square error), mean and maximum tracking error;

- Error of the linear grid model: nodal voltages and branch currents are compared against a posteriori AC power flow. We report the RMSE, mean and maximum error of voltage and currents.

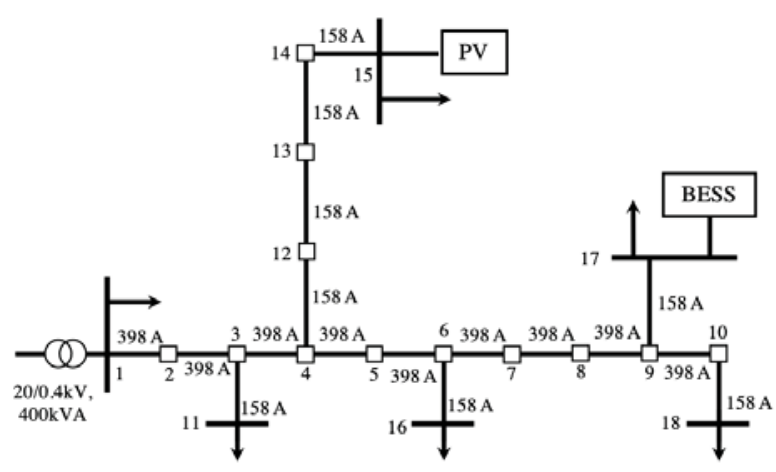

Fig. 1. CIGRÉ low voltage benchmark network [13]

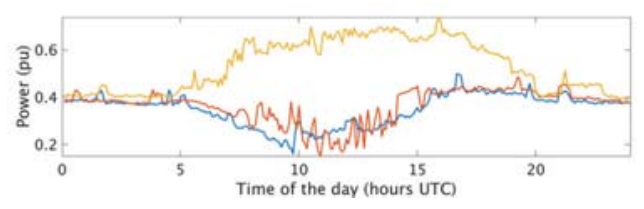

(a) Prosumption at the GCP for three days.

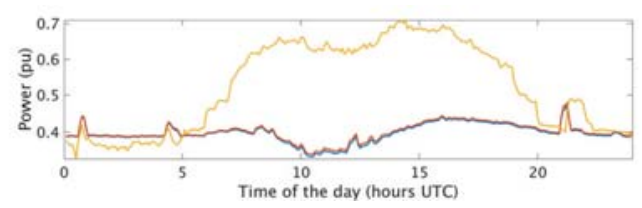

(b) Dispatch plan at the GCP for three days computed using [2].

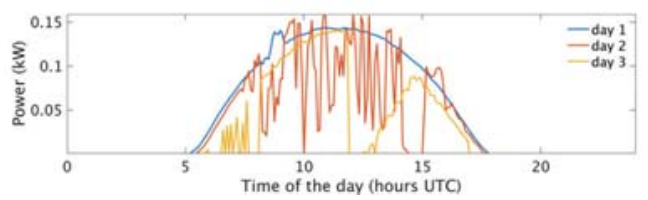

(c) MPP PV forecast for three days.

Fig. 2. (a-c) refers to active power profiles of three days

Table II reports the results of the 3 daily dispatching, where the MPC operates at 5-minute resolution over a shrinking horizon of 24 hours. If ADMM does not converge by the 5minute deadline, a fallback control strategy should be devised. This will be the focus of future work. Above MPC scheme is simulated using the three grid models (denoted by M1, M2, and M3) defined in Sec. II, and the fourth model denoted by M3*, uses M3, but with averaged SCs (averaged w.r.t. next time interval). The maximum ADMM iterations was limited to 100 for above simulations.

Concerning the mean convergence speed, M3* is the fastest. If we compare the maximum simulation time, M3* is 1.1-2.8 times faster than others. All the models perform equally on the tracking error performance. Regarding the voltage error, M2 exhibits the highest error in all the days with a maximum error up to $4 \%$. Also, the mean voltage error is highest with M2. Concerning the current error, the M1, M2, and M3 have the highest errors compared to the ground truth values; it has a maximum current error up to $33 \%$, which is putting the grid 
TABLE II

PERFORMANCE COMPARISON OF DISTRIBUTED MPC CONTROL USING DIFFERENT LINEAR GRID MODELS

\begin{tabular}{|c|c|c|c|c|c|c|c|c|c|c|c|c|c|c|c|c|}
\hline & & \multicolumn{3}{|c|}{ Time (sec) } & \multicolumn{3}{|c|}{ Iterations (\#) } & \multicolumn{3}{|c|}{ Tracking error (kW) } & \multicolumn{3}{|c|}{ Voltage error (pu) } & \multicolumn{3}{|c|}{ Current error (pu) } \\
\hline Day & Model & Min & Mean & Max & Min & Mean & $\operatorname{Max}$ & RMSE & Mean & $\operatorname{Max}$ & RMSE & Mean & Max & RMSE & Mean & Max \\
\hline 1 & M1 & 2 & 60 & 187 & 3 & 36.2 & 100 & 3.6 & -0.33 & 21.4 & $6.1 \mathrm{e}-5$ & $4.4 \mathrm{e}-9$ & $7.4 \mathrm{e}-4$ & $5.5 e-3$ & $-1.3 \mathrm{e}-3$ & $6.0 \mathrm{e}-2$ \\
\hline 2 & M1 & 3.9 & 118 & 265 & 7 & 79 & 100 & 1.68 & -0.28 & 13.2 & $1.5 \mathrm{e}-4$ & $-1.6 e-7$ & $1.8 \mathrm{e}-3$ & $1.6 \mathrm{e}-2$ & $-1 e-2$ & 0.24 \\
\hline 3 & M1 & 2.9 & 121 & 352 & 6 & 77 & 100 & 1.2 & 0.1 & 14.7 & $5 e-5$ & $-5 e-8$ & $5.9 \mathrm{e}-4$ & $5.4 \mathrm{e}-3$ & $-3.0 \mathrm{e}-2$ & 0.1 \\
\hline 1 & M2 & 1.7 & 54 & 242 & 3 & 34 & 100 & 0.42 & -0.21 & 2.4 & $6.3 e-3$ & $2.7 \mathrm{e}-4$ & $4 \mathrm{e}-2$ & $4 \mathrm{e}-2$ & $-3 e-2$ & 0.18 \\
\hline 2 & M2 & 3.7 & 52 & 285 & 6 & 33 & 100 & 0.85 & $-6 e-2$ & 12.71 & $6 e-3$ & $-1.8 \mathrm{e}-4$ & $5 e-2$ & $4 e-2$ & $-2.4 \mathrm{e}-2$ & 0.18 \\
\hline 3 & M2 & 4.6 & 80 & 292 & 8 & 53 & 100 & 1.04 & 0.12 & 12.4 & $7.7 \mathrm{e}-3$ & $-4.1 e-3$ & $5.4 \mathrm{e}-2$ & $4.0 \mathrm{e}-2$ & $-2.0 \mathrm{e}-2$ & 0.23 \\
\hline 1 & M3 & 27 & 48 & 172 & 17 & 33 & 80 & 0.37 & $-6 e-3$ & 2 & $1.4 \mathrm{e}-5$ & $-4.1 \mathrm{e}-6$ & $3.2 \mathrm{e}-4$ & $7.1 \mathrm{e}-4$ & $-7.2 \mathrm{e}-3$ & $3 e-2$ \\
\hline 2 & M3 & 1.7 & 123 & 289 & 2 & 81 & 100 & 1.2 & $-1.6 e-3$ & 13 & $9.8 \mathrm{e}-5$ & $-3.3 e-5$ & $1.6 \mathrm{e}-3$ & $8.2 \mathrm{e}-3$ & $-1.3 e-2$ & 0.12 \\
\hline 3 & M3 & 4.3 & 128 & 273 & 8 & 85 & 100 & 2 & 0.14 & 14.4 & $1.9 \mathrm{e}-4$ & $-4.2 e-5$ & $4.2 \mathrm{e}-3$ & $1.4 \mathrm{e}-2$ & $-2.6 e-2$ & 0.33 \\
\hline 1 & M3* & 18.4 & 41.2 & 150 & 13 & 30.3 & 78 & 0.5 & -0.3 & 2.4 & $4.8 \mathrm{e}-6$ & $-3.3 e-7$ & $1.3 \mathrm{e}-4$ & $5.2 \mathrm{e}-4$ & $-8 \mathrm{e}-3$ & $2.3 \mathrm{e}-2$ \\
\hline 2 & M3* & 1.8 & 35 & 133 & 2 & 17 & 49 & 0.72 & $-4.3 e-2$ & 2.37 & $3.1 \mathrm{e}-6$ & $-1.8 \mathrm{e}-7$ & $5.5 e-5$ & $3.7 e-3$ & $-1.4 \mathrm{e}-2$ & $6.0 \mathrm{e}-2$ \\
\hline 3 & M3* & 2.1 & 25.2 & 126.2 & 2 & 13 & 34 & 0.43 & $-5.3 e-2$ & 1.77 & $1.7 \mathrm{e}-5$ & $-6.0 e-7$ & $4.3 e-4$ & $3.1 \mathrm{e}-3$ & $-3.0 \mathrm{e}-2$ & $6.0 \mathrm{e}-2$ \\
\hline
\end{tabular}

TABLE III

SENSITIVITY OF THE BEST PERFORMING MODEL W.R.T. HORIZON LENGTH

\begin{tabular}{|c|c|c|c|c|c|c|c|c|c|c|c|c|c|c|c|c|}
\hline & Horizon & \multicolumn{3}{|c|}{ Time (sec) } & \multicolumn{3}{|c|}{ Iterations (\#) } & \multicolumn{3}{|c|}{ Tracking error $(\mathbf{k W})$} & \multicolumn{3}{|c|}{ Voltage error (pu) } & \multicolumn{3}{|c|}{ Current error (pu) } \\
\hline Day & hours & Min & Mean & Max & Min & Mean & Max & RMSE & Mean & Max & RMSE & Mean & Max & RMSE & Mean & Max \\
\hline 1 & 12 & 21.4 & 41 & 165 & 12 & 33 & 78 & 0.55 & -0.23 & 4 & $2.7 e-6$ & $-2.1 e-7$ & $9.9 \mathrm{e}-5$ & $4.2 \mathrm{e}-4$ & $-8.1 \mathrm{e}-3$ & $2.2 \mathrm{e}-2$ \\
\hline 1 & 6 & 9.2 & 29 & 127 & 5 & 26 & 71 & 1.28 & -0.16 & 4 & $8.2 \mathrm{e}-6$ & $-1.1 e-6$ & $1.9 \mathrm{e}-4$ & $1.1 \mathrm{e}-3$ & $-5.6 e-3$ & $4 e-2$ \\
\hline 1 & 3 & 3.6 & 29 & 114 & 2 & 33 & 68 & 0.81 & -0.11 & 6.4 & $1.0 \mathrm{e}-5$ & $-1.3 e-6$ & $2.6 \mathrm{e}-4$ & $1.1 \mathrm{e}-3$ & $-4.4 \mathrm{e}-3$ & $3.5 \mathrm{e}-2$ \\
\hline 2 & 12 & 1.6 & 31 & 224 & 2 & 17 & 51 & 0.77 & -0.11 & 4.8 & $4 e-6$ & $-4.4 e-7$ & $6.2 \mathrm{e}-5$ & $4 e-3$ & $-1.4 \mathrm{e}-2$ & $6 e-2$ \\
\hline 2 & 6 & 1.9 & 24.5 & 230 & 2 & 17.6 & 48 & 0.62 & $-1 e-3$ & 4 & $3.4 \mathrm{e}-6$ & $-4.2 e-7$ & $5.1 \mathrm{e}-5$ & $4 e-3$ & $-1.2 \mathrm{e}-2$ & $5.7 e-2$ \\
\hline 2 & 3 & 2 & 22 & 124 & 2 & 24 & 70 & 1.82 & $2.5 \mathrm{e}-2$ & 6 & $6 e-6$ & $-9.8 e-7$ & $1.2 \mathrm{e}-4$ & $3 e-3$ & $-1 \mathrm{e}-2$ & $3 e-2$ \\
\hline 3 & 12 & 2 & 23 & 176 & 3 & 13.3 & 46 & 0.98 & -0.27 & 14 & $1.7 e-5$ & $-5.5 e-7$ & $4.3 \mathrm{e}-4$ & $3.2 \mathrm{e}-3$ & $-3 e-2$ & $6 e-2$ \\
\hline 3 & 6 & 1.9 & 29 & 230 & 2 & 18 & 47 & 0.6 & $9.5 e-2$ & 7 & $1.64 \mathrm{e}-5$ & $-5.5 e-7$ & $4.3 e-4$ & $3.1 \mathrm{e}-3$ & $-3 e-2$ & $6 e-2$ \\
\hline 3 & 3 & 2 & 23 & 175 & 3 & 13.3 & 40 & 0.98 & -0.27 & 14.2 & $1.7 e-5$ & $-5.5 e-7$ & $4.3 e-4$ & $3.2 \mathrm{e}-3$ & $-3 e-2$ & $6 e-2$ \\
\hline
\end{tabular}

TABLE IV

SENSITIVITY OF THE BEST PERFORMING MODEL W.R.T. THE NUMBER OF CONTROLLABLE UNITS

\begin{tabular}{|c|c|c|c|c|c|c|c|c|c|c|c|c|c|c|c|c|}
\hline \multicolumn{2}{|c|}{ BESS } & \multicolumn{3}{|c|}{ Time (sec) } & \multicolumn{3}{|c|}{ Iterations (\#) } & \multicolumn{3}{|c|}{ Tracking error $(\mathrm{kW})$} & \multicolumn{3}{|c|}{ Voltage error (pu) } & \multicolumn{3}{|c|}{ Current error (pu) } \\
\hline \# units & $\mathrm{kWh}$ & Min & Mean & Max & Min & Mean & Max & RMSE & Mean & $\operatorname{Max}$ & RMSE & Mean & Max & RMSE & Mean & Max \\
\hline 2 & 250 & 3.6 & 25 & 254 & 7 & 11 & 72 & 0.42 & 0.11 & 1.5 & $2.8 \mathrm{e}-5$ & $-1.5 e-6$ & $7.4 \mathrm{e}-4$ & $2.3 \mathrm{e}-3$ & $-3.3 e-2$ & $4 \mathrm{e}-2$ \\
\hline 3 & 168 & 7.3 & 32 & 121 & 6 & 7.3 & 25 & 0.5 & 0.11 & 1.7 & $2.2 \mathrm{e}-6$ & $-4.6 e-8$ & $6.4 \mathrm{e}-5$ & $1 \mathrm{e}-3$ & $-3 e-2$ & $3 e-2$ \\
\hline 4 & 125 & 6 & 43 & 132 & 4 & 8.4 & 23 & 0.7 & 0.3 & 2.3 & $2.2 \mathrm{e}-6$ & $-3.2 \mathrm{e}-8$ & $6.6 e-5$ & $8.8 \mathrm{e}-4$ & $-3.2 \mathrm{e}-2$ & $3 e-2$ \\
\hline 5 & 100 & 5.2 & 51 & 149 & 4 & 9 & 22 & 0.62 & 0.25 & 2.4 & $2.5 e-6$ & $-5 e-8$ & $7.9 e-5$ & $9 e-4$ & $-3 e-2$ & $3 e-2$ \\
\hline
\end{tabular}

at the risk of physical damages. M3* is the best performing in terms of the voltage, and current errors with maximum error percentage of $0.043 \%$, and $6 \%$ respectively, and we select it for the next analysis. M3* accounts for probable variations in the future SCs by averaging the SCs of the current and next interval, and thus models the grid constraints more accurately. This also avoids the re-iteration(s) for correcting the SCs.

2) Sensitivity analysis: We present the sensitivity analysis w.r.t. 1) prediction horizon length $T \Delta t$, and 2) number of controllable units in the MPC scheme.

- Sensitivity w.r.t. predictive horizon length: We simulated for shrinking horizon lengths: 3, 6, and 12 hours. We used the same controllable units with equal ratings as before. Table III reports the comparison with horizon length. Results show that the mean convergence time is smaller for shorter horizon length due to fewer decision variables. Also, the model is consistently accurate in terms of modeling errors. The tracking error performance with horizon length will be investigated in future work with particular reference to the possibility of bounding the error of the linearized load flow equalities.

- Sensitivity w.r.t. the number of controllable units: Table IV reports the computational performance (for day 3) when increasing the number of controllable units. We simulate distributed BESSs with equal size among them and total energy capacity as the case before, and placed at nodes 11, 13, 14, 16, and 17. From the table, we observe that even if the number of BESS units is increased up to 5 , the increase in convergence time and the number of iterations is not significant, thus denoting good scalability property. Also, it is noteworthy to mention that voltage and current errors are consistently small.

\section{Output profiles}

Fig. 3 shows the simulation results of the MPC algorithm with the grid model M3* for day 1 . In particular, Fig. 3a refers to the performance of the dispatch plan tracking: it can be observed that the prosumption at the GCP highly differs from the dispatch plan due to the incorrect forecast of the 


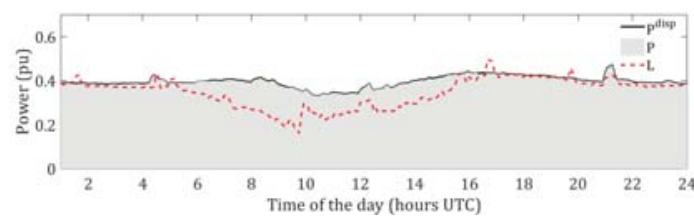

(a) Dispatch plan (black), prosumption realization (shaded area), uncontrolled active power flow at the GCP (dashed red).

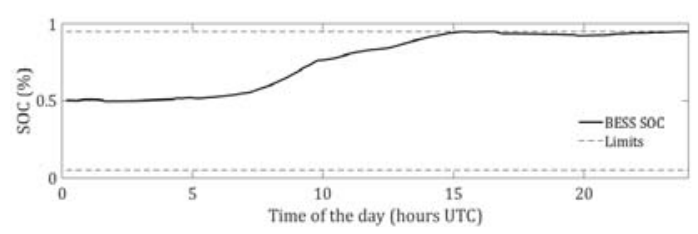

(b) Battery power injection (upper panel), and battery SOC evolution and respective limits (bottom panel).

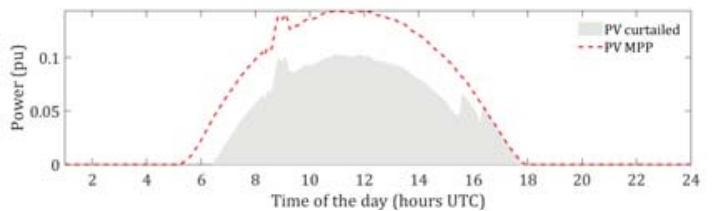

(c) Curtailed PV (shaded area), and theoretical MPP PV (in dashed red)

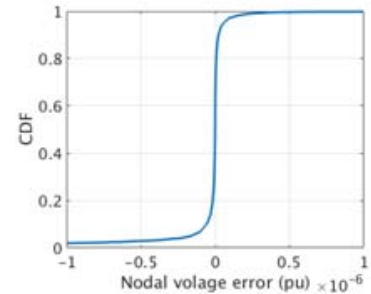

(d) CDF plot of the voltage error

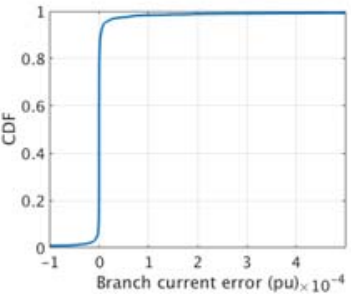

(e) CDF plot of the current error
Fig. 3. MPC operation using M3* on day 1.

prosumption. The MPC is able to control the BESS and PV to achieve a successful dispatch. Fig. $3 b$ shows the BESS SOC evolution. Fig. $3 c$ shows the active power set-points of the PV resource: the curtailed PV is shown in shaded grey, and maximum possible PV generation in dashed red. From Fig $3 \mathrm{c}$, it can be seen that the control scheme starts curtailing PV generation from the beginning of the day till hour 16. This helps to i) keep BESS SOC within its operational limits, and ii) accurately track the dispatch plan. Finally, figures $3 \mathrm{~d}$ and $3 \mathrm{e}$ report the cumulative distribution functions of the nodal voltage and branch current modelling errors w.r.t. the $\mathrm{AC}$ power flow computations. They show that the voltage and current errors, for the $99 \%$ of the estimations, are in the range [-7.2e-6 pu, 3.8e-7 pu] and [-3.9e-4 pu, 1e-3 pu] respectively.

\section{Conclusions}

We compared the performance of a grid-aware MPC to dispatch the operation of resources in a distribution system, when the underlying grid model (based on sensitivity coefficients) was updated according to different policies: once per day considering static injections, once per day considering dynamic point predictions of the injections, and dynamically updated during the day. The dispatch plan was computed with the objective of restoring a suitable level of flexibility in the available energy storage resources while accounting for the stochasticity in the prosumption. We simulated for two resources: PV and BESS. The BESS losses was modeled by integrating it into the network admittance matrix. The performance of the control strategy was evaluated w.r.t. convergence speed, tracking error and modeling errors. The analysis showed that the model, where the linear transformation parameters are updated dynamically using the averaged sensitivity coefficients, outperforms others in terms of convergence speed and accuracy in the modeling of grid constraints. We showed that the the linear grid model is exact w.r.t. the load-flow computations, when it is updated dynamically. Further, the sensitivity analysis of the best performing model showed that i) the algorithm can be made faster by reducing the horizon length without compromising its performance, and ii) the algorithm is scalable w.r.t. the number of controllable units.

\section{REFERENCES}

[1] M. Abu Abdullah, K. Muttaqi, D. Sutanto, and A. Agalgaonkar, "An effective power dispatch control strategy to improve generation schedulability and supply reliability of a wind farm using a battery energy storage system," IEEE Trans. Sustainable Energy, vol. 6, 2015.

[2] F. Sossan, E. Namor, R. Cherkaoui, and M. Paolone, "Achieving the dispatchability of distribution feeders through prosumers data driven forecasting and model predictive control of electrochemical storage,' IEEE Trans. Sustainable Energy, vol. 7, no. 4, pp. 1762-1777, Oct 2016.

[3] R. Gupta, F. Sossan, E. Scolari, E. Namor, L. Fabietti, C. Jones, and M. Paolone, "An admm-based coordination and control strategy for pv and storage to dispatch stochastic prosumers: Theory and experimental validation," in 2018 Power Syst. Comput. Conf., June 2018, pp. 1-7.

[4] L. Gan et al., "Exact convex relaxation of optimal power flow in radial networks," IEEE Trans. Autom. Control, vol. 60, no. 1, pp. 72-87, 2015.

[5] M. Nick, R. Cherkaoui, J.-Y. Le Boudec, and M. Paolone, "An exact convex formulation of the optimal power flow in radial distribution networks including transverse components," IEEE Trans. Autom. Control, vol. 63, no. 3, pp. 682-697, 2018.

[6] J.-H. Teng, "A direct approach for distribution system load flow solutions," IEEE Trans. Power Del., vol. 18, no. 3, pp. 882-887, 2003.

[7] P. Fortenbacher, A. Ulbig, and G. Andersson, "Optimal placement and sizing of distributed battery storage in low voltage grids using receding horizon control strategies," IEEE Trans. Power Syst., vol. 33, no. 3, pp. 2383-2394, 2018.

[8] K. Christakou, J.-Y. LeBoudec, M. Paolone, and D.-C. Tomozei, "Efficient computation of sensitivity coefficients of node voltages and line currents in unbalanced radial electrical distribution networks," IEEE Trans. Smart Grid, vol. 4, no. 2, pp. 741-750, 2013

[9] M. Paolone, J.-Y. Le Boudec, K. Christakou, and D.-C. Tomozei, "Optimal voltage control processes in active distribution networks," The Institution of Engineering and Technology-IET, Tech. Rep., 2015.

[10] S. Boyd et al., "Distributed optimization and statistical learning via the alternating direction method of multipliers," Foundations and Trends in Machine Learning, vol. 3, 2011.

[11] B. He, H. Yang, and S. Wang, "Alternating direction method with selfadaptive penalty parameters for monotone variational inequalities," $J$. Optimization Theory applicat., vol. 106, no. 2, pp. 337-356, 2000

[12] E. Stai, L. Reyes, F. Sossan, J.-Y. Le Boudec, and M. Paolone, "Dispatching stochastic heterogeneous resources accounting for grid and battery losses," IEEE Transactions on Smart Grid, vol. 9, no. 6, pp. 6522-6539, 2018.

[13] C. T. F. C6.04.02, "Benchmark systems for network integration of renewable and distributed energy resources," Cigre' International Council on large electric systems, Tech. Rep., July 2009.

[14] M. Pignati et al., "Real-time state estimation of the epfl-campus medium-voltage grid by using pmus," in Conf. Innovative Smart Grid Technologies (ISGT), no. 1-5, 2015. 